Audrey RoIG

Aspirante du Fonds de la Recherche Scientifique-FNRS

Université Libre de Bruxelles - ULB

Centre de recherche LaDisco

Plateforme internationale de recherche Gramm-R

\title{
À la recherche du lien entre les constructions averbales du type Autant de têtes, autant d'avis et les structures corrélatives isomorphes proportionnelles verbales.
}

\section{Introduction}

La corrélation, à envisager comme la mise en relation de deux séquences où l'une suppose l'autre et réciproquement, peut revêtir plusieurs formes en français contemporain. À côté de tournures particulières en ce qu'elles ne présentent à priori pas de marqueur corrélatif, il est possible de corréler deux séquences par le recours précisément à des termes corrélatifs, tels que :

(a) des connecteurs primaires ${ }^{1}$ ou secondaires ${ }^{2}$ fonctionnant en marqueur unique ( $a$ mesure quelde, au fur et à mesure quelde, comme, ainsi que, en corrélation avec, en fonction de, en parallèle, parallèlement,...);

(b) des connecteurs primaires ou secondaires non identiques fonctionnant en marqueurs doubles (autant...que, aussi...que, moins...que, plus...que, si...que, non seulement...mais, celui-ci...celui-là, le premier...le deuxième, l'un ...l'autre,...) ;

(c) des connecteurs primaires ou secondaires identiques fonctionnant en marqueurs binomiaux (ni...ni, et...et, plus...plus, autant...autant, soit...soit, tantôt...tantôt, tel...tel,...).

Dans le cadre de cette contribution, seule la catégorie (c) retiendra notre attention. Elle regroupe les corrélatives isomorphes $(\mathrm{CI})$, soit les structures dont les prédications sont ouvertes chacune par un même marqueur corrélatif (ou un corrélatif de sens contraire).

Par-delà l'apparente homogénéité morphosyntaxique des dénommées « CI », l'analyse $\mathrm{du}$ discours de plusieurs grammaires d'usage révèle la coexistence de deux groupes sémantiques de CI, qui sont directement fonctions du contenu idiosyncrasique des marqueurs. Le premier groupe rendrait essentiellement compte d'une " opération de disjonction » (Charaudeau 1992) et explicite un choix, une alternative (Eluerd 2008, Grevisse \& Goosse 2011) (les CIA ; i.e. 1, 2, 3), exception peut-être pour ni...ni qui développerait également la conjonction sémantique (Charaudeau 1992), bien que tous

Sont dits connecteurs primaires les conjonctions et les prépositions (cf. Wilmet 1997, 2007 ; Van Raemdonck \& Detaille 2011).

2 Sont qualifiés de secondaires les connecteurs de type adverbial, adjectival (qualifiant ou quantiqualifiant, mais non quantifiant) ou pronominal, termes dont la fonction prototypique n'est pas la liaison de séquences ( $c f$. Van Raemdonck \& Detaille 2011). 
les linguistes ne s'accordent pas sur ce dernier point (cf. Mouret 2007). Le second groupe rassemble les corrélatives dites comparatives (Chevalier et al. 1990 ; Riegel et al. 2004 ; Grevisse \& Goosse 2007, 2011) (les CIP ; i.e. 4, 5, 6), bien que la notion de comparaison interroge (Cappeau \& Savelli 1995) et couvre d'ailleurs un spectre plus large de structures ${ }^{3}$ que celles étudiées ici.

(1) Je m'engageais dans mon premier métier officiel : un métier ni vraiment choisi, ni refusé (Gault V., Le corps incertain, 2006, p. 17)

(2) Et soit intentionnellement, soit par réflexe involontaire, elle proféra une grossièreté à l'égard de la défunte mère de Hampâté. (Bâ A., Amkoullel, l'enfant peul, 1991, p. 58)

(3) Tantôt bon enfant, et tantôt bête fauve. (Chandernagor F., L'Enfant des Lumières, 1995, p. 321)

(4) autant les cours et les intérieurs de ferme nous rassurent, autant les intérieurs ouvriers me troublent déjà, avec leurs lits défaits, leur abandon, leur précarité locative, leur noircissement (Guyotat P., Formation, 2007, p. 92)

Plus j'y pense, plus il me semble que Léo était d'une stupidité très caractérisée mais qui n'était pas sans charme - du moins pour moi. (Duras M., Cahiers de la guerre et autres textes, 2006, p. 79-80)

(6) Tel était le système anglais, tel est actuellement le système américain. (MendèsFrance P., CEuvres complètes. 5. Préparer l'avenir. 1963-1973, 1989, p. 723)

Toutefois, l'étude des siamoises en plus...plus, etc. disposerait d'un autre dénominateur commun d'ordre sémantique, à savoir la proportion. Dans ces structures, en effet, « la quantité ou degré exprimé dans une proposition augmente ou diminue au fur et à mesure qu'augmente ou diminue la quantité ou le degré exprimé dans l'autre » (Sanchez Lopez 2010 : 135). Ériger la proportion au rang de trait définitoire des corrélatives plus...plus, autant...autant et tel...tel reste néanmoins une pratique interrogeable. Comme l'a remarqué Allaire (1982), la proportion s'applique en effet davantage aux structures plus...plus (et variantes combinatoires) qu'aux CI en autant ou en tel, en proie surtout à la comparaison. Les deux étiquettes se montrent donc problématiques, mais, en l'absence d'une nomenclature plus juste, nous privilégierons présentement l'appellation de corrélatives isomorphes " proportionnelles " (CIP) pour ce qu'elle ne s'applique pas aux constructions du type autant...que, etc. Seule cette deuxième famille de corrélatives isomorphes sera abordée dans cette contribution, famille qui, au demeurant, semble constituée tantôt de structures verbales, tantôt de constructions averbales. Si elles sont souvent traitées pêlemêle (section 1), nous verrons néanmoins ci-dessous, après étude des propriétés respectives de l'une et l'autre construction (sections 2 et 3 ) que ces secondes ne sauraient être les dérivées syntaxiques des premières (section 4), et qu'il importe en conséquence de ne pas les considérer comme des manifestations de CIP verbales elliptiques. Ces structures entretiendraient en effet plus de liens avec les CI non marquées du style Tu fais un pas, tu es un homme mort qu'avec les traditionnelles CIP verbales (section 5).

\footnotetext{
3 En effet, la dénomination désigne également les énoncés du type Pierre mange plus que Louis par
} exemple. 


\section{1) Les CIPa sont des CIPv elliptiques}

À l'intérieur de l'ensemble des CIP - groupe aux dénominations hétérogènes ${ }^{4}$-, il est possible de distinguer deux sous-familles, suivant que les séquences corrélées présentent ou non un verbe conjugué à temps fini. L'on parlera dans le premier cas de structures CIP verbales ( $\mathrm{CIPv})$; dans le second, de CIP averbales (CIPa). Perçues comme de simples CIPv ellipsées, les CIPa sont généralement écartées des travaux sur les corrélatives proportionnelles en linguistique. La tendance est identique dans les grammaires d'usage. Ainsi, Tomassone (2002: 173) mêle indistinctement les deux constructions, verbales et averbales. Grevisse \& Goosse (1993, 2007, 2011 : § 263) traitent également les $\mathrm{CIPa}$ de la même manière que les $\mathrm{CIPv}$, pointant pour seule différence l'absence de verbe dans ces premières.

En particulier, des comparatifs en corrélation indiquent un rapport proportionnel ou inversement proportionnel : Plus on est puissant, plus on est tenu d'être juste (Taine, Orig. de la Fr. contemp., t. III, p. 262). - Plus tu veux, moins tu peux (R. Rolland, Jean-Chr., t. I, p. 190). - Autant la Normandie progresse, autant la Bretagne est en décadence (Michelet, Mer, I, 3). - Plus l'armagnac est vieux, meilleur il est. (...)

Les sous-phrases sont averbales : Tel père, tel fils (prov.). - Autant d'hommes, autant de cochons (Zola, Terre, IV, 2). - Autres temps, autres moeurs (prov.). (Grevisse \& Goosse $2011: \S 263)$

Dans leur Grammaire méthodique du français (2004), Riegel, Pellat \& Rioul n'opèrent pas davantage de distinction entre les deux structures qu'il convient d'étudier ensemble. Plus engagés toutefois, les auteurs recourent à présent au terme d'ellipse pour qualifier l'absence de formes verbales.

\section{Constructions parallèles}

Chacune des deux propositions a son introducteur spécifique, et le système exprime soit l'équivalence quantitative, soit la variation proportionnelle: autant... autant...; plus... plus... ; moins... moins... ou encore plus... moins ; moins... plus ... : Plus on est de fous, plus on rit-Moins je le vois, (et) mieux je me porte. Ou elliptiquement: Autant de têtes, autant d'avis - Beaucoup de paille, peu de grain. (Riegel et al. 2004 : 516)

La mention de l'adverbe elliptiquement sous-tend l'hypothèse que les segments verbaux, s'ils ne sont pas réalisés phonologiquement, le sont cependant syntaxiquement $^{5}$ (Busquets \& Denis 2001 ; Dagnac 2008). Les CIPv et les CIPa seraient

4 Si Cappeau \& Savelli (1995) et, par la suite, Stage (2009) les nomment «siamoises», d'autres linguistes leur préfèrent l'appellation de "corrélatives comparatives》 ou celle de "comparatives corrélatives » (Moline 1996; Borsley 2004; Abeillé \& Borsley 2006; Laurens 2007 ; Bertocchi \& Maraldi 2010 ; Sanchez Lopez 2010), tandis que d'autres encore recourent plus volontiers à l'étiquette de "Comparative Conditionals» comme c'est le cas par exemple pour McCawley (1988) et Beck (1997).

5 C'est là l'une des deux approches théoriques concurrentes sur la façon de concevoir l'ellipse, l'autre définissant l'ellipse en termes d'anaphore et de complément nul. Cette deuxième thèse ayant été préalablement mise à mal par Busquets \& Denis (2001) puis par Dagnac (2008), et puisque nous avons fait état par ailleurs des raisons pour lesquelles cette approche ne convient pas dans le cas des CIPa (cf. Roig à paraitre), nous ne proposerons ici que la lecture de l'existence d'éléments syntaxiquement structurés mais non réalisés phonologiquement. Voir à ce propos Hadermann, 
donc deux constructions identiques dans leur essence. L'analyse approfondie de ces tours permet pourtant d'objecter en partie à cette thèse. La constitution puis l'analyse d'un corpus ${ }^{6}$ de CIP incitent en effet à traiter différemment les CIPv et les CIPa, qui ne partagent pas toujours les mêmes propriétés morphosyntaxiques et sémantiques, ni ne répondent pareillement aux tests auxquels ces structures sont généralement soumises.

\section{2) Les CIP « verbales »}

Les CIPv, pour commencer, affichent généralement une parfaite équivalence morphosyntaxique (Allaire 1982) : la répétition du marqueur en tête de séquence, la famille de corrélatifs sélectionnée, la conjugaison du verbe à un mode personnel, identique (7) ou non (8), dans les deux prédications ${ }^{7}$ en sont autant d'exemples :
Autant notre oncle Illiouscha se réjouissait d'être, militairement parlant, écarté, autant notre père ressassait l'humiliation. (Schreiber B., Un silence d'environ une demi-heure, 1996, p. 223)
Cela se passait il y a longtemps. Je suis redevenu journaliste et j'ai rejoint la BBC lorsqu'elle a été fondée. Quand Cort est-il mort ? » N'est-il pas étrange que, plus on vieillit, plus on soit sensible à la mort des autres ? (Pears I., La chute de John Stone, trad. de G.-M. Sarotte, (s.1.), Belfond, 2009)

Les CIPv sont toujours constituées de deux prédications minimum, indissociables (Cappeau \& Savelli 1995 ; Stage 2009). Leur ordre est immuable sous peine d'altérer le sens de l'énoncé (9) en raison du rapport d'implication (Beck 1997, Abeillé \& Borsley 2006) ou d'ordre linéaire (Melis 1994) qui oriente la lecture de la majorité de ces constructions. Ouverte par une forme comparative (Stage 2009) de portée extraprédicative (Hadermann et al. à paraitre(a)), chaque séquence est elle-même formée d'un sujet et d'un prédicat, et la réduction ou l'effacement du verbe dans l'une des deux séquences, voire dans les deux, rend la CI agrammaticale (10) :

(9) $\quad(a) \neq(b)$, car :

(a) Ici, plus on approche de la victoire, plus Mme Bordes se vide, mais elle se lèvera. (Duras M., Cahiers de la guerre et autres textes, 2006, p. 211)

(b) plus Mme Bordes se vide, plus on approche de la victoire *Autant notre oncle Illiouscha se réjouissait d'être, militairement parlant, écarté, autant notre père ø l'humiliation.

En termes de propriétés morphosyntaxiques, les CIPv tolèrent pour la plupart :

- l'insertion de et aux confins des deux séquences (Allaire 1982, Abeillé \& Borsley

Pierrard, Roig \& Van Raemdonck, Ellipses et fragments. Morceaux choisis, Bruxelles, PIE-Peter Lang, à paraitre.

6 Les exemples constitutifs de notre corpus sont majoritairement issus de la base de données Frantext, des Archives du Monde diplomatique et d'occurrences trouvées sur les forums de discussion du net pour l'écrit, et du Corpus de français parlé parisien pour l'oral (Branca-Rosoff S., Fleury S., Lefeuvre F., Pires M., Discours sur la ville. Corpus de Français Parlé Parisien des années 2000 (CFPP2000)).

7 Dans ce cas, il semblerait que seule la forme verbale de la deuxième séquence (Préd2) puisse être au subjonctif, tandis que le verbe de la première séquence serait ou à l'indicatif (asymétrie des modes), ou au subjonctif également (symétrie modale). 
2006, Stage 2009),

(11) (a) Plus j'ai d'argent, et plus l'idée du manque d'argent est angoissante (avoir un million sur mon compte en banque me paralyse toujours). (Guibert H., Le Mausolée des amants, 2001, p. 196)

(b) Autant il disait m'aimer et autant il me faisait du mal psychologiquement. (internaute, 34 ans, posté le 25 octobre 2011, consulté le 15 mars 2012) Car plus je connais, plus je veux connaître, plus je suis disposé pour connaître, plus je convoite le bien d'autrui et plus je le pille et plus je m'engraisse à le dévorer. (Saint-Exupéry A. de, Citadelle, 1944, p. 623)

- la focalisation d'un groupe commun aux deux parties de la CI (Abeillé \& Borsley 2006 ; Stage 2009) et, à plus forte raison, la focalisation de la première prédication (Préd1) dans son intégralité (Hadermann et al. 2010 : 231),

(13) (a) C'est un auteur que plus on lit -, plus on apprécie -. (Abeillé \& Borsley 2006)

(b) C'est une personne qu'autant ils célèbrent -, autant ils briment. (d'après Mertens P., Les Eblouissements, 1987, p. 243).

(14) Elle a peur, certes, elle a peur de voir galvauder, abîmer inutilement son trésor. Mais c'est plus elle est sûre de ce qu'elle tient que plus elle a peur de le hasarder... (Rivière J., À la trace de Dieu, 1937, p. 36).

- l'extraction symétrique mais non l'asymétrique (Abeillé \& Borsley 2006) :

(15) (a) C'est un auteur que plus on lit -, plus on apprécie-. (Abeillé \& Borsley 2006)

(b) *C'est un auteur que plus on lit ses livres, plus on apprécie -. (ibid.)

(c) *C'est un auteur que plus on lit - ; plus on aimerait le connaître. (ibid.)

- l'inversion du sujet clitique dans chacune des séquences simultanément, d'après Abeillé \& Borsley (2006), bien que notre corpus ne comporte que très peu d'attestations du genre. L'on trouve cependant plus facilement des cas de distorsions dans des productions plus ou moins spontanées d'internautes sur des forums de discussion :

(16) (a) Plus vite sera t'elle partie, plus vite reviendra t'elle. (homme, 17 ans, posté le 30 décembre 2006, consulté le 15 mars 2012)

(b) Son autre main se glisse de manière autoritaire sous ma jupe et m'agrippe la fesse. Ces manières autoritaires sont assez nouvelles pour moi mais je comprends aussi qu'il n'y pas lieu de trainer en préliminaires, au plus vite aurons-nous assouvi notre envie de sexe, au moins on a de risque de se faire surprendre. (internaute, 48 ans, posté le 17 décembre 2010, consulté le 28 avril 2011)

- ou la présence d'un pronom clitique dans la première prédication (Préd1) dont le sujet nominal de la prédication 2 (Préd2) serait coréférent (Abeillé \& Borsley 2006),

(17) (a) Tel j'apparaissais dans ces pages, tel j'étais (Roubaud J., La Boucle, 1993, p. 259)

(b) > Tel Robin apparaissait dans ces pages, tel il était.

(c) $>$ Tel il apparaissait dans ces pages, tel Robin était.

- l'enchâssement, bien que les CIPv paraissent résister à la réduplication de la conjonction que de sorte que le subordonnant ne puisse être trouvé en tête de chaque séquence (18) : * « ... que + Corrélatif + Préd1, que + Corrélatif + Préd 2 » (Abeillé \& Borsley 2006; Hadermann et al. 2010), en dépit de l'attestation hapaxique rencontrée chez Céline (19) : 
(18) Je crois que plus le récit est proche dans le temps avec l'expérience qui le suscite, plus je suis à l'aise dans l'écriture. (Guibert H., Le Mausolée des amants, 2001, p. 527)

(19) [... s'en plaignaient jamais cependant parce qu'il est bien entendu qu'un soldat est aussi brave qu'insouciant, et grossier plus souvent qu'à son tour, et que plus il est grossier et que plus il est brave. (Céline L.-F., Voyage au bout de la nuit, 1932, p. 91)

En ce qui concerne le registre de la sémantique, les CIPv se particulariseraient par le principe de covariation qu'elles engendrent (Stage 2009, Sanchez Lopez 2010). Ainsi, toute modification du degré dans la première prédication déclenche un changement d'ordre quantitatif ou qualitatif dans la deuxième séquence. Une quantité stable en Préd1, en revanche, implique une stabilité, quantitative ou qualitative, dans la Préd2. De l'avis de Stage, «la construction permet [ainsi] à l'interlocuteur d'inférer soit une relation conditionnelle soit une relation causale » $(2009: 254)$. La teneur du propos vise particulièrement les CIP du type plus...plus tandis que le discours ne vaut pas tellement pour les structures autant...autant ou tel...tel. Dans les trois exemples cités ci-dessous (ex. 20-22), en effet, l'on ne saurait identifier un quelconque lien de causalité ou quelque forme de condition. À contrario, plus...plus rend facilement l'une et/ou l'autre circonstance(s).

(20) Autant, en effet, il méprisait la presse à sensation et autant il avait de respect pour une liberté formelle de la presse. (Toller E., Une jeunesse en Allemagne, 1974, p. 116)

(21) Autant il rend l'existence inexistante, et autant il rend l'inexistence existante. (Nasar J., Le rien en architecture, l'architecture du rien, 2011, p. 49)

(22) Telle est l'immanence dans la transcendance et telle est la transcendance dans l'immanence. (Lagarde M., Le livre des haltes, 2002, p. 274) ${ }^{8}$

(23) Et, sans doute, plus on a souffert, plus il s'est déroulé d'événements graves, plus on s'attache. (Havet M., Journal 1919-1924, 2005, p. 419)

\section{3) Les CIP « averbales »}

Homogène en apparence, l'ensemble des $\mathrm{CIPa}^{9}$ rassemble en réalité des structures variées, qui peuvent être organisées en trois catégories. La première réunit les tours de loin les plus fréquents, à savoir les CIPa formées d'un « adjectif quantifiant ${ }^{10}+\mathrm{N}$ » (type 1), tantôt ayant l'allure de comparatifs, tantôt prenant la forme de négatifs, comme dans les exemples (24a), (24b) et (24c). Le deuxième tiroir de corrélatives averbales se

8 À la lecture de l'exemple (22) se pose la question de savoir si la structure tel...tel, lorsqu'elle ouvre des prédications verbales, bénéficie encore du statut de CI. Le principe de co-relation qui régit les CI semble en effet amoindri dans ces tours, et il n'est pas impossible que l'on ait affaire à une juxaposition de deux phrases simples.

9 Nous laissons volontairement de côté les structures en apparence hybrides comme Un verre de cognac et je conquiers le monde, qui sont en réalité à ranger du côté des corrélatives non marquées du type $T u$ dis un mot et je te renvoie d'où tu viens. Cette classification éclaire en outre la difficulté de restituer parfois avec précision le matériel élidé dans ces premières constructions.

10 Simple ou complexe ; désormais abrégé $\mathrm{Adj}^{\mathrm{Qt}}$. 
caractérise par la réunion de structures composées d'un " adjectif (quanti)qualifiant ${ }^{11}(+$

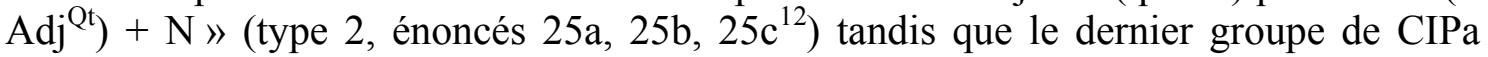
concentre les constructions « $(\grave{a})+l e+$ adverbe » (type 3 ), comme l'illustrent (26a) et (26b).

(24) (a) Plus de participations, plus de commentaires, plus d'avis (internet)

(b) plus de pont praticable, plus de contact avec la rive droite (Rambaud P., La Bataille, 1997, p. 105)

(c) $\varnothing$ Chose promise, $\varnothing$ chose due. (Prov.)

(25) (a) Tel père tel fils (Lanzmann J., La Horde d'or, 1994, p. 207)

(b) Autres temps, autres mœurs. (Prov.)

(c) Meilleurs les élèves, meilleures les notes (Laurens $2007: 92$ )

(26) (a) Au plus, au mieux. (Oral)

(b) Le plus, le mieux. (Oral)

D'après Grevisse \& Goosse, les constructions au (plus) ou le (plus) constitueraient des variantes régionales ou populaires des CI standards (2011: § 986). L'agrammaticalité de la CI *Plus, mieux, c'est-à-dire de la corrélative dépourvue de la particule le (et de a), interroge cependant l'assimilation de la totalité de ces tours aux productions régionales et populaires.

Par ailleurs, le corpus qui sert de base à cette étude ne comporte pas d'occurrence telle que celle présentée par Laurens (2007) (i.e. 25c), qui serait pourtant un tour fréquemment attesté en anglais ${ }^{13}$ (Borsley 2004). Si l'absence de ce genre d'exemples dans notre corpus n'implique pas son inexistence, nous ne les traiterons néanmoins pas dans cette contribution, de la même manière que nous ne développerons pas ici les cas de (25a) et (25b) qui mériteraient une attention particulière. Quant aux exemples (26a) et (26b), ils semblent être des cas particuliers de CIPv réduites à leur plus haut degré. Ces CIPa ne fonctionnant pas comme les structures de type 1, nous ne les étudierons pas dans cette contribution.

Les $\mathrm{CIPa}^{14}$ de type 1 ne partagent pas toutes les propriétés formelles des CIPv et certains de ces manquements sont facilement imputables au caractère "elliptique » des CIPa. Comme les CIPv, les CIPa sont constituées de minimum deux séquences ${ }^{15}$, qui témoignent chacune d'une équivalence formelle tantôt parfaite (autant de têtes, autant d'avis), tantôt partielle (Beaucoup de paille, peu de grains). Indissociables ${ }^{16}$ sans perte

11 Désormais abrégé $\mathrm{Adj}{ }^{\mathrm{Q}}$.

12 Les énoncés (25a) et (25c) ne sont cependant pas tout à fait assimilables : l'on ne saurait faire correspondre exactement aux structures $\left\langle\mathrm{Adj}^{\mathrm{Ql}}+\mathrm{Adj}^{\mathrm{Qt}}+\mathrm{N} »(\mathrm{ex} .25 \mathrm{c})\right.$ l'énoncé Tel père, tel fils, composé d'un quantiqualifiant et d'un nom.

13 Par exemple : «The more intelligent the students, the better the marks » (Borsley 2004).

14 Dès à présent, il convient d'entendre par $C I P a$ les seules structures corrélatives averbales de type 1.

15 Les CIPa sont rarement composées de plus de deux séquences, mais cette éventualité n'est pas écarter puisque ce type d'occurrences est attesté : Autant de visages et de corps, autant d'ombres, autant de fantômes (Pontalis J.-B., Un homme disparaît, 1996, p. 134).

16 Exception faite pour les structures aujourd'hui figées et devenues proverbiales, les séquences des CIPa sont moins solidaires que celles des CIPv. Les séquences des structures averbales restent en effet grammaticalement correctes et sémantiquement recevables lorsqu'elles sont considérées individuellement (i.e. <Il n'y a> plus de pont! <Je voudrais> plus d'histoires!), bien que l'on perde 
de l'effet corrélatif et irréductibles, les prédications sont immobiles en raison d'un « effet iconique de linéarité » (Melis $1994: 110)$ ou du «cadre d'implication logique » (Hadermann et al. 2010 : 227) formé par la Préd1. Les CIPa ne comportent forcément jamais de verbe conjugué à un temps fini, et les circonstants cadratifs ou extraprédicatifs semblent être absents de ces tours si l'on se réfère à notre corpus. Toutes se composent au contraire de deux GN mis en relation, éventuellement déterminés par une sous-phrase relative. En ce sens, les CIPa étonnent par leur apparente simplicité syntaxique.

Dans le prolongement de ces observations, l'on peut encore remarquer que chacune des séquences qui composent la CI est ouverte par un $\mathrm{Adj}^{\mathrm{Qt}}$ que l'on a vite fait de désigner marqueur eu égard aux constructions verbales. À la différence toutefois des CIPv, ce potentiel terme corrélatif n'est pas toujours de forme comparative, puisque les locutions pas de, beaucoup de, etc. peuvent occuper la position initiale caractéristique de ces structures corrélatives. Aussi, alors qu'elle est extraprédicative dans les CIPv classiques, la portée de ce marqueur est ici systématiquement intraprédicative ${ }^{17}$.

autant d'images, autant de possibles. (Pontalis J.-B., L'enfant des limbes, 1998, p. 17)

D'autres aspects distinguent les CIPa de type 1 de leurs homologues verbales, notamment l'impossibilité d'insérer et entre les deux séquences (*Préd1 et Préd2) sans perdre l'effet corrélatif (28) ou celle d'enchâsser la structure (29). Les CIPa résistent également à certaines manipulations syntaxiques issues de tests distributionnalistes, telles que la focalisation de la première séquence (30) ou l'extraction d'une partie de la structure corrélative, souvent réduite déjà à son plus simple apparat, ce qui confère généralement au tour un caractère gnomique.

(28) *autant d'images et autant de possibles.

(29) *je pense qu'autant d'images, autant de possibles.

(30) *C'est autant d'images qu'autant de possibles.

Les CIPv et les CIPa n'adoptent donc pas toujours le même comportement, et les propriétés sur lesquelles elles diffèrent ont parfois trait à l'essence même des structures corrélatives isomorphes.

Au niveau sémantique, de même, si les deux constructions approchent l'une de l'autre, elles ne peuvent être confondues. En effet, comme les CIPv donnent lieu à une covariation entre le degré ou l'orientation dicté(e) dans l'une et l'autre séquence, toute information quantitative livrée dans la première séquence influence la quantité de la deuxième. À l'inverse de leurs apparentes homologues verbales, cependant, les structures averbales permettraient systématiquement «à l'interlocuteur d'inférer soit une relation conditionnelle soit une relation causale » (Stage $2009: 254$ ), alors que nous avons vu que ce n'était pas toujours le cas pour les CIPv. Les structures averbales approchent en cela les constructions CI non marquées hypothétiques du genre Tu fais un

dès lors l'effet corrélatif. Une prédication verbale issue d'une $\mathrm{CIPv}$ ne peut en revanche exister seule : * Plus il mange.

17 Voir infra, fin de la section $4:$ Les CIPa, des CIPv réduites? 
pas, tu es un homme mort, qu'étudient notamment Borillo (2010) ou Corminbœuf (2008). Cela étant, les CIPa ne sauraient être tout à fait assimilées à ces constructions corrélatives non marquées puisque, si l'insertion d'un conjonctif tel que et aux confins des deux prédications est autorisée dans ces corrélatives verbales, le procédé demeure impossible pour les CIPa, fait déjà signalé supra.

L'ensemble de ces constats invite à repenser la nature du lien qui unit les CIP verbales et averbales : les CIPa ne seraient-elles pas des corrélatives verbales ellipsées ?

\section{4) Les $\mathrm{CIPa}$, des CIPv réduites?}

Les observations faites jusqu'alors n'apportent pas d'éléments de réponse clairs. La question est d'autant plus obscure qu'il semble complexe de donner une analyse structurelle non ambigüe des prédications d'une CIPa. "Autant de têtes, autant d'avis » sera en effet perçu soit comme la double association d'un « adverbe corrélatif $+d e+$ $\mathrm{N} »(31 \mathrm{a})$, soit comme la réduplication de deux $\mathrm{SN}$ respectivement formés d'un « $\mathrm{Adj}{ }^{\mathrm{Qt}}$ complexe (adverbe non corrélatif $+d e)+\mathrm{N} »(31 \mathrm{~b})$.

(31) (a) autant + de + têtes

(b) autant de + têtes

La première des deux analyses, celle qui met en exergue l'existence d'un marqueur corrélatif, plaide en faveur d'un rapprochement des CIPa et des CIPv. La deuxième, au contraire, parce qu'elle nie l'existence même du marqueur corrélatif adverbial qui ouvre les séquences d'une CIPv, invite à cloisonner les deux types de CIP ; dans cette optique, verbales et averbales n'entretiendraient plus de lien aujourd'hui. L'observation de certains tours en latin classique montre cependant que ce ne fut pas toujours le cas, au minimum pour les structures autant...autant :

quot hominum linguae $<$ sunt $>$, tot nomina deorum $<$ sunt $>$ (Cicéron, in Rousseau $2005: 51)$

(34) quot orationum genera esse diximus totidem oratorum reperiuntur. (Cicéron, in Fry $2005: 262)$

Le couple de corrélatifs quot...tot..., indéclinables, ouvre les parties constitutives de la corrélative en (32) et (33) comme en (34), indépendamment de la propriété verbale ou averbale de la CI. L'ellipse du verbe esse est une pratique courante à l'époque, et l'omission de sunt en (32) et en (33) semble n'avoir aucun impact sur la manière d'analyser l'énoncé. Fry (2005) relève toutefois la facilité qu'ont quot...tot... de s'associer à des substantifs, alors que ces corrélatifs sont souvent remplacés par d'autres pour des raisons morphologique et phonologique lorsqu'il s'agit d'une prédication complète : «les pluriels, surtout verbaux, ont souvent plus de substance phonique que n'en ont les singuliers ", écrit-elle (2005: 263). Parce que la présence du verbe a tendance à occulter les corrélatifs quot...tot..., d'autres adverbes de corrélation sont utilisés plus couramment pour l'ouverture d'une prédication verbale, notamment quam...tam, quo (magis)...eo (magis), quanto...tanto. L'on pourrait penser qu'une grammaticalisation différente prit ensuite le pas et accentua le clivage entre les CIPa et 
les CIPv amorcé en latin classique pour la construction autant...autant et, par extension, plus...plus.

Il semblerait en effet que sous l'effet du temps, les adverbes latins faisant office de corrélatifs se soient grammaticalisés, donnant lieu aujourd'hui à des marqueurs de corrélation syntaxiquement figés, toujours placés en tête des prédications ${ }^{18}$. Aux couples "subordonnants quo, quanto \& adverbes relatifs $e o$, tanto" se sont ainsi petit à petit substitués deux adverbes isomorphes dans les CIPv, et la grammaticalisation progressive de ces adverbes a emporté avec elle toute trace physique d'hypotaxe comme le donnent à voir les exemples suivants :

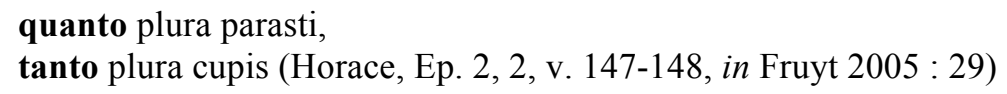

quanto plura parasti, tanto plura cupis (Horace, Ep. 2, 2, v. 147-148, in Fruyt 2005 : 29)

(Plus tu as acquis de richesses, plus tu en désires davantage).

Quant je plus vos acointe, et je vos truis plus fols (De Boron R., Merlin, XIIIe s., 42, 28, in Bonnard \& Régnier $1989: 225$ )

(Plus je vous fréquente, plus je vous trouve fou).

Toutes voies a verité celle proprieté singuliere que, quant plus est foulee, tant plus se ressourt, et sont les commencements poignans a soustenir et durs, mais son yssue est aggreable et fructueuse (Chartier A., Le Quadrilogue invectif, 1422, p. 43)

Car d'autant qu'un homme court plus hastivement hors du chemin, d'autant plus se recule-il hors de son but, et à ceste cause est plus misérable. (Calvin J., Institution de la religion chrestienne : livre troisième, 1560, p. 248)

Bien que l'hypothèse demanderait à être plus largement vérifiée par l'étude d'un corpus qui couvre la période allant du latin classique au $\mathrm{XVI}^{\mathrm{e}}$ siècle $^{19}$, il n'est pas interdit de penser que les CIPa latines auraient elles aussi été concernées par le processus de grammaticalisation; mais l'ellipse du verbe dans l'Antiquité déjà, aurait conféré un autre statut auxdits adverbes, les spécialisant très vite dans la formation $\mathrm{d}^{\mathrm{Adj}}{ }^{\mathrm{Qt}}$ complexes ou "quantifiants composés" (Wilmet 2003: 178) au même titre que beaucoup de, plus de, assez de, etc. Une telle pensée se voit confortée par deux faits :

- l'attestation de CIPa dans leur forme définitive dès le début du XVI ${ }^{\mathrm{e}}$ siècle déjà :

(39) (a) car, comme l'on dit, autant de testes, autant d'opinions (de La Mothe Le Vayer F., Dialogues faits a l'imitation des anciens..., 1506, p. 21)

18 Hadermann, Pierrard, Roig \& Van Raemdonck écrivent d'ailleurs à ce sujet : « les CI illustreraient un cas de grammaticalisation, soit achevé, soit toujours en cours, dont les composants des marqueurs ne seraient plus seulement des adverbes dans leur emploi mais également des connecteurs, sans qu'ils puissent être reversés à cette classe au premier chef. Ce serait ce processus que traduirait précisément le figement progressif de la position du marqueur, variable encore aux $\mathrm{XVII}^{\mathrm{e}}$ et $\mathrm{XVIII}{ }^{\mathrm{e}}$ siècles. Cette manière d'envisager les CI sied par ailleurs si l'on envisage l'évolution de ces structures sur le long terme : l'hypotaxe, de mise en latin classique, continue d'être marquée en ancien puis en moyen français, voire même de temps à autre en français préclassique. Cette observation [...] n'interdit pas [...] de penser à une prise en charge des valeurs que véhiculaient autrefois les autres termes de la locution corrélative (quanto...tanto [...]) par le seul item corrélatif restant aujourd'hui, à savoir plus. Le principe à l'œuvre serait alors le même que celui identifié pour l'évolution des marqueurs de négation ne...pas, ne...plus, etc., où la polarité du premier élément (ne), sa charge sémantique, s'est progressivement mais significativement déplacée sur le second terme (pas, plus) » (à paraitre(a)).

19 Notre corpus ne comprend en effet aucune attestation de CIPa de type I avant le XVI ${ }^{\mathrm{e}}$ siècle. 
(b) Mais telles gens sont du nombre de ceste ignorance populaire qui n'ont rien de certain et sont tant aveuglez qu'ilz suyvent en jugement de toutes choses la premiere opinion qu'ilz ont, desquelz on dit : Autant de testes, autant d'opinions. (Vienne $\mathrm{Ph}$. de, Le Philosophe de court, 1548, p. 166)

- la possibilité de créer une CIPa à partir de n'importe quel Adj ${ }^{\mathrm{Qt}}$, même assez de ou un peu de dont les constructions corrélatives sont néanmoins plus rares :

(40) (a) Assez de sang, assez de plasma. (internet, consulté le 15 mars 2011)

(b) Assez d'hommes, assez de choix. (internet, forum sur les Histoires de Coeur, consulté le 15 mars 2011)

(41) Un peu de soleil, un peu de baume au cœur (internet, consulté le 15 mars 2011)

Dans l'absolu, la confirmation de cette hypothèse rendrait absurde toute volonté de traiter sans distinction, aujourd'hui, les CIPa et les CIPv, étant donné que ces structures n'entretiendraient plus de lien d'un point de vue synchronique.

C'est l'option des quantifiants complexes que choisissent Grevisse et Goosse dans Le bon usage en faisant de autant de un déterminant indéfini occasionnel (1993 : 927), citant alors cet exemple : «autant d'hommes, autant d'avis. " (ibid.; 2011 : 805). À l'heure actuelle, autant de, plus de, pas de, etc. fonctionneraient donc comme des quantifiants qui, lorsqu'ils ouvrent deux séquences successives mises en relation, donnent lieu à une structure corrélative.

D'autres arguments favorisent la thèse d'une grammaticalisation différente pour les CIPa et les CIPv. En anglais d'abord, les constructions verbales et averbales ne sont pas les mêmes :

(42) More money, more problems.

(43) The more John eats, the more he pays. (Culicover \& Jackendoff $2005: 504$ )

En français, ensuite, l'on peut trouver une CIPa ouverte par un adverbe de quantité nulle (pas, plus), ce qui est impossible pour les CIPv. Toutes les CIPa ne trouvent donc pas de CIPv équivalentes :

(44) Pas de bras, pas de chocolat. (Hadermann et al. $2010: 234$ )

(45) plus de pont praticable, plus de contact avec la rive droite (Rambaud P., La Bataille, 1997, p. 105).

(46) *Pas John mange, pas il paie.

*plus John mange, plus il paie (où plus est adverbe de négation).

Rapprocher pas de et plus de des autres quantifiants composés est une pratique qui a fait couler beaucoup d'encre. Si Gaatone (1992) fait de pas de un "groupe NEG + de », Van de Velde (1994) préfère simplement traiter pas de comme beaucoup de; de même, Wilmet range sous la rubrique «quantifieur $+d e$ », les associations $A d v .+d e$ (assez de, beaucoup de, ...) et les $N+$ de (pas de, point de, nombre de, quantité de,...) (2003 : 178). Lefeuvre revient quant à elle sur cette catégorisation, refusant de mêler pas de aux autres formes quantifiantes complexes. Elle motive son choix par l'impossibilité pour pas de de figurer en position syntaxique de sujet (48) et le fait que le groupe ne fonctionne pas toujours en binôme solidaire (49). Ainsi, conclut-elle, "pas de 
est proche des autres quantifieurs mais sans constituer pour autant un déterminant» (1999a : 297).

*Pas de vent ne soufflait. (Muller 1991 : 291, in Lefeuvre 1999a : 297)

(49) Comprenez-nous, on n'a pas vu de femmes depuis des années. (Veil S., Une vie, 2007, p. 83)

Deux exemples, dont l'un trouvé dans la Grammaire critique du français (2010), permettent cependant d'objecter à l'observation de Lefeuvre (1999a) :

(50) Pas de spectacle vaut mieux qu'un mauvais spectacle. (Wilmet $2010: 432$ ).

Pas d'économies implique pas de dépenses (Van Raemdonck, oral)

Postuler l'existence d'un prédicat ellipsé du type «<ne pas voir $>$ de spectacle » ou « < ne pas disposer> d'économies » résoudraient toutefois les difficultés posées par (50) et (51) - bien que pas de soit alors syntaxiquement éclaté -, et l'exemple cité ne contreviendrait plus par conséquent à l'observation de Lefeuvre. Admettons néanmoins que pas de n'ouvre jamais de séquences de fonction sujet ; cela ne constitue pas pour autant un critère suffisant à sa marginalisation. Il est facile d'observer que tous les Adj ${ }^{\mathrm{Qt}}$ ne peuvent pas être trouvés dans toutes les situations. Les quantifiants bipolaires $d u$ et des, par exemple, n'ouvrent normalement pas un SN en position de sujet s'ils sont d'extensité maximale, c'est-à-dire en lecture universelle. Exception faite en lecture thétique (Roig 2010). À l'instar de cette observation, nous pouvons postuler qu'il serait possible de trouver l'une ou l'autre situation exceptionnelle pour pas de. Ces analyses nous invitent à ranger pas de avec les autres $A d j^{Q t}$ complexes ou quantifiants composés.

Une grammaticalisation différente semble ainsi être à l'origine de la cloison qui sépare les CIPv et les CIPa. Les anciens adverbes corrélatifs, devenus aujourd'hui des Adj ${ }^{\mathrm{Qt}}$ complexes, entrent désormais dans la composition des CIPa sous la forme suivante :

$$
\begin{aligned}
& \text { Autant de têtes } \\
& \text { Autant de }+ \text { têtes } \\
& (\text { Adverbe }+d e+\varnothing)+\mathrm{N}
\end{aligned}
$$

Le test de la dislocation motive la présence de l'article $\varnothing$ dans la chaine de termes constituant les quantifiants complexes. Parce que la désagrégation du groupe adverbe + de ne peut se faire sans modification de cet article (Wilmet 2010:423), il est important de prévoir une telle position dans la formation des quantifiants composés.

(53) (a) Pierre a montré pas mal de courage. (Wilmet $2010: 423$ )

(b) Du courage, Pierre en a montré pas mal. (ibid.)

Et si les anciens adverbes sont effectivement devenus des $\mathrm{Adj}^{\mathrm{Qt}}$ complexes dans le cas des $\mathrm{CIPa}$, chaque prédication de la corrélative devrait répondre de la même manière au test de la dislocation, ce qui semble être le cas.

(54) (Il n'y a) pas de chocolat.

(55) Du chocolat, il n'y en a pas.

(56) (Il y a) autant de têtes.

(57) Des têtes, il y en a autant. 
Lefeuvre (2000) voit en ces anciens adverbes négatifs ou d'intensité des appuis, c'est-àdire des marqueurs de prédication, "parce qu'ils jouent un rôle par rapport à la prédication et qu'ils semblent marquer, d'une fonction prédicative, les mots sur lesquels ils portent. » (Lefeuvre 1999b). Ils sont «fréquemment indispensables » (ibid.) et « leurs prototypes sont concernés par la quantification ou bien par l'intensité » (ibid.). Ainsi donc, si les CIPa sont, d'un point de vue synchronique, des structures qui mettent en relation deux SN dont l'un implique l'autre et réciproquement, il n'est pas proscrit de voir dans ces structures l'expression d'une ellipse. Le «prototype » des CIPa toutefois, pour reprendre le terme de Tamba-Mecz (1983 : 152), ne serait pas à chercher du côté des CIPv. Le sens des énoncés comme plus de pont praticable, plus de contact (Rambaud) donne à penser que le prototype des CIPa serait davantage une structure existentielle du type « $<I l\left(n^{\prime}\right) y a>[\mathrm{X}],\left\langle i l\left(n^{\prime}\right) y a>[\mathrm{Y}] »\left(\mathrm{v}\right.\right.$. Roig à paraitre $\left.{ }^{20}\right)$. Cette hypothèse se voit par ailleurs corroborée par la fonction qu'assigne Lefeuvre aux marqueurs de prédications, celle d'actualiser le substantif dans sa fonction prédicative (Lefeuvre 2000).

\section{5) Les Adj ${ }^{\text {Qt }}$ complexes, des marqueurs corrélatifs ?}

L'Adj ${ }^{\mathrm{Qt}}$, de prime abord, s'il n'est certainement pas un connecteur primaire, n'a pas en soi davantage vocation de fonctionner en tant que connecteur secondaire. La présence de l'adverbe dans la composition du quantifiant composé pourrait toutefois laisser à penser que le quantifiant véhiculerait encore intrinsèquement la valeur de marqueur corrélatif. Cette façon de concevoir va pourtant à l'encontre de la thèse de la grammaticalisation, même si cette hypothèse conforterait la possibilité pour les $\mathrm{Adj}{ }^{\mathrm{Qt}}$ complexes d'être à la base de structures corrélatives. Force est de reconnaitre néanmoins que d'autres structures corrélatives averbales du type ne disposent pas d'élément adverbial dans leur constitution, comme Chose promise, chose due, où les substantifs sont actualisés par des $\mathrm{Adj}^{\mathrm{Qt}} \varnothing$, ou Un phare, un port, voire encore Pas de nouvelles, bonnes nouvelles - qui illustre de surcroit un cas de distorsion morphologique entre les deux quantifiants. Exception faite pour pas de, ces énoncés ne comportent en effet que des Adj ${ }^{\mathrm{Qt}}$ de base. En conclusion, l'adverbe présent dans la forme adjectivale ne peut pas avoir la valeur de terme corrélatif. Il serait sinon proscrit de trouver une structure corrélative avec pour seules entrées des Adj ${ }^{\mathrm{Qt}}$ et les substantifs qu'ils actualisent. De là procèdent deux possibilités : soit il revient à $\mathrm{l}^{\prime} \mathrm{Adj}{ }^{\mathrm{Qt}}$ simple d'exercer la fonction de marqueur corrélatif - l'adjectif quantifiant devenant, par ce fait, un connecteur secondaire -, soit les CIPa se caractérisent par l'absence de marqueurs

20 La question de la nature du matériel élidé a fait l'objet d'une contribution antérieure intitulée " "Autant de têtes, autant de tableaux différents peut-être" : une structure corrélative isomorphe proportionnelle averbale ?». Dans celle-ci, nous présentions en outre une série d'arguments qui permettaient d'objecter partiellement aux conceptions traditionnelles de l'ellipse dans le cas des CIPa. Nous expliquions également pourquoi ces structures corrélatives averbales ne peuvent davantage être regardées comme des séquences fragmentaires, ni même être pleinement assimilées aux phrases averbales de Lefeuvre (1999). 
corrélatifs, comme c'est le cas dans les structures telles que $<$ Tu fais $>$ un pas de plus, et il va t'arriver malheur.

La possibilité de nier l'Adj ${ }^{\mathrm{Qt}}$ dans certaines structures corrélatives averbales mais non dans toutes, plaiderait pour la coexistence de deux nouvelles catégories de CIPa : celles dont l'Adj ${ }^{\mathrm{Qt}}$ peut être précédé d'un polaire négatif tel que pas et dans lesquelles il n'y aurait alors pas de marqueur corrélatif (58); et celles dont les prédications ne peuvent pas être niées - à l'instar ici des CIPv - et qui seraient éventuellement ouvertes par un marqueur corrélatif (59). En réalité, ces dernières ne sont pas davantage composées de marqueurs corrélatifs que celles du précédent groupe en vertu de la possibilité de trouver les séquences introduites par autant de, pas de, plus de, etc. en emploi unique, c'est-à-dire sans qu'elles soient dupliquées. Partant, la présence d'un marqueur corrélatif dans la première séquence mais non dans la seconde prédication de l'exemple (60) questionnerait particulièrement.

(58) (a) Pas assez de fumée, pas assez de sensations. Je voudrais beaucoup plus de fumée sans avoir à tirer sans arrêt (internet, consulté le 15 mars 2011)

(b) Pas assez de pluie $=$ pas assez de fruits. (internet, consulté le 15 mars 2011)

(c) Pas beaucoup de pluie, peu de bonnes récoltes.

(59) (a) *Pas pas de bras, pas pas de chocolat.

(b) *Pas autant de têtes, pas autant d'avis.

(c) *Pas plus de personnes, pas plus d'opinions.

(60) (a) Beaucoup de pluie, peu de bonnes récoltes.

(b) Pas beaucoup de pluie, peu de bonnes récoltes.

(c) *Beaucoup de pluie, pas peu de bonnes récoltes.

Dans l'un et l'autre cas en effet, les séquences d'une CIPa ne deviennent corrélatives qu'en association avec une autre séquence semblable, le fonctionnement de ces constructions différant de ce point de vue des CIPv : les constructions pas de bras / plus de pont / beaucoup de blé / etc. peuvent en effet fonctionner en autarcie tout en restant porteuses de sens et grammaticalement correctes ; les prédications verbales, en revanche, nécessitent la réalisation de la seconde séquence pour que la construction demeure acceptable. Autrement dit, il est impossible d'avoir *Plus il court, sans que soit énoncée successivement la prédication impliquée par l'action de courir plus : plus il court, plus il a soif. La réunion des deux prédications, dans les cas des structures averbales, ne devient pertinente que si elle correspond à une volonté du locuteur de faire jaillir un effet de sens corrélatif. Autrement dit, des CIPa nait une corrélation strictement praxéologique alors que les $\mathrm{CIPv}$ entretiennent un rapport de corrélation syntaxique et sémantique avant d'être pragmatique. De ce point de vue, les CIPa approcheraient à nouveau les énoncés verbaux du type Tu m'embrasses encore, et c'est mon pied dans les pompons ! (Corminbœuf 2008 : en ligne) plutôt que les CIPv, soit les structures corrélatives sans marqueurs. Dans ces CI verbales, en effet, les deux prédications peuvent être attestées sans être unies syntaxiquement (mais le sens est alors propre dans chaque séquence) sans que cela n'occasionne de difficultés. Ce n'est qu'une fois assemblées qu'elles établissent une corrélation. 
Ce constat ouvre la porte à deux autres : 1) si la structure profonde des CIPa est effectivement une structure existentielle hypothétique du type $\langle i l$ (n') y $a>$, il n'y aurait en effet pas de marqueur visible pour ouvrir les séquences corrélées ; 2) l'absence de marqueur corrélatif tangible dans certaines CIPa favorise le rapprochement de ces structures avec les CI non marquées telles que celles étudiées par Borillo (2010) ou Corminbœuf (2008), ce qui a pour effet d'accentuer encore le fossé entre les structures CIPa et CIPv. Partant de cette observation, un parallèle pourrait éventuellement être remarqué entre l'absence de marqueurs corrélatifs et l'expression de la condition. Les $\mathrm{CIPa}$ comme les CIv conditionnelles non marquées véhiculent en effet toujours cette relation sémantique. En conséquence, l'on pourrait postuler l'existence d'un marqueur «si» sous-jacent dans l'une et l'autre construction, connecteur qui ferait éventuellement partie du matériel élidé. À contrario, il est également pensable que le connecteur hypothétique non réalisé phonologiquement ne soit pas non plus réalisé syntaxiquement, mais qu'il soit tout au plus le fruit d'un effet de sens particulier à ces structures. Cette idée est étayée par la possibilité notamment pour les CIPv de porter cette même relation sémantique asymétrique (v. Mouret 2007), alors qu'elles refusent catégoriquement d'être précédées du morphème si sans que la grammaticalité de l'énoncé en soit atteinte. La présence du connecteur oblige en effet au déplacement du marqueur corrélatif antéposé. Ce faisant, la portée initialement extraprédicative du corrélatif devient intraprédicative, et il y a rhématisation de l'adverbe quantitatif initialement thématisé (cf. Van Raemdonck et al. 2011).

(61) $\quad<$ S'il y a $>$ plus de ponts praticables, $<$ il y a $>$ plus de contacts.

(62) $<\mathrm{Si}>$ tu fais un pas, tu es un homme mort.

(63) (a) $\quad *<$ Si $>$ plus il mange, plus il grossit.

(b) Si il mange plus, on grossit plus.

Irrésolue présentement, cette question devrait faire l'objet d'une autre étude pour recevoir toute l'attention qu'elle mérite.

\section{Conclusion}

Par cette contribution, nous entendions ébrécher un mur érigé par la tradition, et permettre ainsi la sortie du placard de structures oubliées ou rangées trop hâtivement avec les autres CIP verbales. Le rattachement des CIPa aux corrélatives isomorphes proportionnelles verbales du type Plus il mange, plus il grossit est en effet une pratique tentante mais en réalité erronée. Si les deux structures sont, pour certaines constructions, d'origine identique, les deux types de CIP n'ont toutefois résolument pas bénéficié du même processus de grammaticalisation. Ainsi, alors que le terme placé en tête de prédication dans les $\mathrm{CIPv}$ prend la forme d'un adverbe corrélatif de quantité ou d'intensité, dans le cas des CIPa, plus, moins, autant, etc. constituent avec l'Adj ${ }^{\mathrm{Qt}}$ auquel il a fini par s'associer, un $\mathrm{Adj}^{\mathrm{Qt}}$ complexe, au même titre que beaucoup de, peu de, pas de, etc. Par le phénomène de grammaticalisation, l'adverbe y a donc délaissé son statut de marqueur corrélatif. Les CIPa se particularisent dès lors par une simplicité morphosyntaxique accrue par l'absence physique de tout marqueur corrélatif. Ces 
constructions averbales approchent de ce point de vue les corrélatives comme celles traitées par Borillo et Corminbœuf (C'est beaucoup d'argent, mais ce n'est pas cher, Sempé), bien que les deux structures, à nouveau, ne sauraient être complètement amalgamées. Parmi d'autres questions, il resterait encore à analyser le degré de ressemblances entre les deux structures non marquées, comme celle de savoir s'il y a effectivement absence de marqueur dans ces dernières constructions ou s'il $\mathrm{y}$ apparaitrait plutôt des marqueurs corrélatifs ø.

Dans le prolongement de ce travail, il serait intéressant d'étudier la question du mode de liaison de prédications opérant dans les CIPa. Si l'on peut penser que les séquences d'une CIPv entretiennent un lien syntaxique ou de subordination, éventuellement faible (Abeillé \& Borsley 2006, Orlandini \& Poccetti 2009, Sanchez Lopez 2010) ou de coordination (Allaire 1982, Culicover \& Jackendoff 2005), il n'est pas certain que les structures isomorphes averbales répondent de la même façon à l'indexation des structures aux catégories subordination/coordination, ni même que le mode de liaisons de prédications à l'œuvre dans Tel père, tel fils (CIPa type 2) soit le même que dans Pas de bras, pas de chocolat (CIPa type 1) ou dans Le plus, le mieux (CIPa type 3).

\section{Bibliographie :}

ABeIllé A., Borsley R., 2006, «La syntaxe des corrélatives comparatives en anglais et en français ", Faits de Langues, 28, p. 21-33.

Allaire S., 1982, Le modèle syntaxique des systèmes corrélatifs. Étude en français moderne, Thèse présentée à l'Université de Rennes II, Service de reproduction des thèses, Université de Lille III.

BECK S., 1997, "On the semantics of Comparative Conditionals », Linguistics and Philosophy, 20, p. 229-271.

BertocCHI A., MARALDI M., 2010, «Latin comparative correlatives and scalarity », in HADERMANn P. et al. (dir.), Approches de la scalarité, Genève, Droz, p. 113-134.

Bonnard H., RÉGNier Cl., 1989, Petite grammaire de l'ancien français, Paris, Magnard.

BORILlO A., 2010, "La corrélation hypothétique et la construction parataxique », in BÉGUELIN M.-J. et al. (dir.), La parataxe : Structures, marquages et exploitations discursives, t. 2, Berne, Peter Lang, p. 3-26.

Borsley R., 2004, « An approach to English Comparative correlatives », in MülLER S. (éd.), Proceedings of the HPSG, Katholieke Universiteit Leuven, Stanford, CSLI Publications, p. 70-92.

Busquets J., Denis P., 2001, «L'ellipse modale en français: le cas de devoir et pouvoir », Cahiers de Grammaire, 26, p. 55-74.

Cappeau P., Savelli M.-J., 1995, «Corrélation ne vaut pas comparaison », Faits de langues, 5, p. 175-182.

Charaudeau P., 1992, Grammaire du sens et de l'expression, Paris, Hachette Éducation. 
Chevalier J.-C., Benveniste Cl.-B., Arrivé M., Peytard J., 1990, Grammaire du français contemporain, Paris, Larousse.

Corminbeuf G., 2008, " "Tu m'embrasses encore, et c'est mon pied dans les pompons !" Comment construit-on le sens ? », Discours, 3, en ligne.

Culicover P., Jackendoff R., 2005, Simpler Syntax, Oxford, Oxford University Press.

DAGNAC A., 2008, «L'ellipse modale en français : arguments pour une ellipse du TP »,

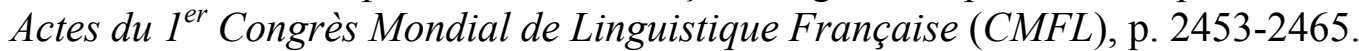

ELUERD R., 2008, Grammaire descriptive de la langue française, Paris, Armand Colin.

FRY C., 2005, « Corrélatifs catégoriels et cognition : talis... qualis et les autres », in DE CARVAlho P., LAMBert F. (dir.), Structures parallèles et corrélatives en grec et en latin, Actes du Colloque de Bordeaux, septembre 2002, Saint-Etienne, Publications de l'Université de Saint-Etienne, p. 255-266.

FRUYT M. 2005, «La corrélation en latin: définition et description », in P. De Carvalho, F. Lambert (dir.), Structures parallèles et corrélatives en grec et en latin, Actes du Colloque de Bordeaux, septembre 2002, Saint-Etienne, Publications de 1'Université de Saint-Etienne, p. 17-44.

GAATONE D., 1992, «De négatif entre la syntaxe et la sémantique. Réflexions sur quelques propriétés du déterminant de », Langue française, 94, p. 93-103.

GREvisSE M., $1993^{13} / 2007^{14} / 2011^{15}$, Le bon usage, éd. revue par GoOSSE A., Paris/Louvain-la-Neuve, De Boeck/Duculot.

Hadermann P., Pierrard M., Van Raemdonck D., Wielemans V., 2010, « Les structures corrélatives : pour une inscription dans les sous-systèmes parataxe / hypotaxe et coordination / subordination », in BÉGUELIN M.-J. et al. (éd.), La parataxe: Structures, marquages et exploitations discursives, t. 2, Berne, Peter Lang, p. 219-239.

Hadermann P., Pierrard M., Roig A., VAN RAEMdonck D., à paraitre(a), «Les structures corrélatives en plus...plus : le point de vue des marqueurs de liaison ", Actes du colloque Corrélation : aspects syntaxiques et sémantiques, Université de Genève, 1-2-3 septembre 2011, Genève, Droz.

Hadermann P., Pierrard M., Roig A., Van Raemdonck D. (dir.), à paraitre(b), Ellipse \& Fragment. Morceaux choisis, Bruxelles, PIE Peter Lang.

LAURENS F., 2007, Analyse et formalisation des types de phrases averbales du français, mémoire de maitrise ss la dir. d'ABEILlé A., Université Paris Diderot - Paris 7, consultation en ligne: http://www.linguist.univ-paris-diderot.fr/ flaurens, consulté le 16 mars 2011.

LEFEUVRE F., 1999(a), La phrase averbale en français, Paris, L'Harmattan.

LEFEUVRE F., 1999(b), "Les "marqueurs de prédication" dans la phrase averbale en français », Verbum, 21, p. 429-438.

LEFEUVRE F., 2000, «Exemple de la phrase averbale existentielle», Le Français Moderne, LXVIII, 2, p. 191-201.

MCCAWLEY J., 1988, "The Comparative Conditional in English, German and Chinese ", Proceedings of the Fourteenth Annual Meeting of the Berkeley Linguistics Society, 14, p. 176-187. 
Melis L., 1994, «La typologie des subordonnées circonstancielles et les comparatives ", Travaux de linguistique, 27, p. 97-111.

Moline E., 1996, "Pierre est-il aussi intelligent que Paul ? Syntaxe des comparatives corrélatives quantifiantes ", Cahiers d'études romanes, 8, p. 165-205.

MoURET F., 2007, Grammaire des constructions coordonnées : coordinations simples et coordinations à redoublement en français contemporain, thèse de doctorat, Université Paris 7, en ligne.

Orlandini A., PoccetTi P., 2009, «Corrélation, coordination et comparaison en latin et dans les langues italiques », Langages, 174, p. 53-66.

Riegel M., Pellat J.-C., Rioul R., 2004, Grammaire méthodique du français, Paris, PUF.

RoIG A., 2010, «Existe-t-il une lecture universelle pour les $\mathrm{SN} d u N$ en position syntaxique de sujet? Comparaison avec les SN des $N$ en pareille situation », communication au IX Congrès International de Linguistique française, Madrid, 24-25-26 novembre 2010.

RoIG A., à paraitre, " "Autant de têtes, autant de tableaux différents peut-être" : une structure corrélative isomorphe proportionnelle averbale ? ", in HADERMANN P., Pierrard M., RoIG A., VAN RAEMdONCK D. (dir.), Ellipse \& Fragment: morceaux choisis, Bruxelles, P.I.E. Peter Lang.

Rousseau A., 2005, «Les structures corrélatives en latin: syntaxe et sémantique, origines dans les langues indo-européennes anciennes ", in DE CARVALHO P., LAMBERT F. (dir.), Structures parallèles et corrélatives en grec et en latin, Actes du Colloque de Bordeaux, septembre 2002, Saint-Etienne, Publications de l'Université de Saint-Etienne, p. 45-62.

SANCHEZ LOPEZ C., 2010, "Scalarité et corrélation: syntaxe et sémantique des corrélatives comparatives en espagnol », in HADERMANN P. et al. (éds), Approches de la scalarité, Genève, Droz, p. 135-168.

Stage L., 2009, «Les constructions siamoises. Étude sur les comparatives corrélatives », Hermès, 43, p. 251-294.

TAMBA-Mecz I., 1983, «L'ellipse, phénomène discursif et métalinguistique », Histoire, Épistémologie, Langage, 5, p. 151-157.

VAN DE VeldE D., 1994, « Le défini et l'indéfini », Le Français moderne, 62, p. 11-35.

VAn RAEMdonck D., Detaille M., 2011, Le sens grammatical. Référentiel à l'usage des enseignants, avec la coll. de Meinertzhagen L., Bruxelles, P.I.E. Peter Lang.

WiLMET M., $1997^{1} / 2003^{3} / 2010^{5}$, Grammaire critique du français, Bruxelles, de BoeckDuculot.

WiLMET M., 2007, Grammaire rénovée du français, Bruxelles, de Boeck-Duculot. 